\title{
ALIMENTAÇÃO DE JUVENIS DA SARDINHA PAPUDA Triportheus flavus (OSTEICHTHYES, CHARACIDAE) NO PERIODO DE CHEIA, NO LAGO CAMALEÃO (AMAZONAS, BRASIL)
}

\author{
Miriam Adriana ALVÁN-AGUILAR ${ }^{1,2}$, Yamile BENAION ALENCAR ${ }^{2}$, Elsa RODRIGUES HARDY ${ }^{4}$, \\ Fred CHU-KOO ${ }^{1,3}$
}

1 Universidad Científica del Perú - UCP. Facultad de Ciencias del Medio Ambiente y Biotecnología. Escuela Profesional de Ecología. Avenida Abelardo Quiñónez Km. 3.5. Iquitos, Perú. E-mail: miriam.alvan@gmail.com

2 Instituto Nacional de Pesquisas da Amazônia. Coordenação de Pesquisas em Entomologia. Caixa Postal 478, Manaus, AM. Brasil.

3 Instituto de Investigaciones de la Amazonía Peruana - IIAP. Programa para el Uso y Conservación del Agua y sus Recursos AQUAREC. Carretera Iquitos - Nauta, Km. 4.5. Iquitos, Perú. E-mail: fchuk20@gmail.com

4 Instituto Nacional de Pesquisas da Amazônia. Coordenação de Pesquisas em Biologia Aquática. Laboratorio de Plancton. Caixa Postal 478, Manaus, AM. Brasil.

\section{RESUMO}

A sardinha papuda, Triportheus flavus, é um peixe de porte mediano que alcança os $25 \mathrm{~cm}$ quando adulto. Na Amazônia Central, esta espécie é migradora, de reprodução total e constitui um importante recurso para as pescarias da região. No presente trabalho, estudou-se a composição da dieta de 50 exemplares juvenis da sardinha papuda Triportheus flavus (55.4 9.9 mm CP) no Lago Camaleão (estado de Amazonas, Brasil). Os peixes foram coletados das zonas com abundância de macrófitas aquáticas, durante o período de cheia de 1999. A dieta foi composta de vinte e oito itens alimentares, sendo os insetos os mais importantes com nove ordens identificadas. Registrou-se também, a presença de ácaros, cladóceros, copépodos, aranhas, camarões, escamas de peixes e sementes. Conclui-se que no Lago Camaleão, T. flavus quando juvenil é um peixe onívoro e possui um amplo espectro alimentar, sendo que sua dieta é composta principalmente de invertebrados como Formicidae e Homoptera.

PALAVRAS CHAVE: Triportheus flavus, dieta, items alimenticios, insetos, Lago Camaleão.

\section{FEEDING OF JUVENILE SARDINA Triportheus flavus (OSTEICHTHYES, CHARACIDAE) IN THE HIGH-WATER SEASON, IN THE CAMALEÃO LAKE, (AMAZONAS, BRAZIL)}

\begin{abstract}
The sardina, Triportheus flavus, is a medium-size fish that can reach up to $25 \mathrm{~cm}$ of total length when adult. In the Central Amazon, it is a migratory fish, total spawner and represents an important natural resource for regional fisheries. In the current work, the feeding habits of 50 juvenile individuals of sardina Triportheus flavus (55.4 9.9 $\mathrm{mm} \mathrm{SL}$ ) were studied in Camaleão Lake (Amazonas State, Brazil). Fish were collected from areas covered by macrophytes during the high- water season of 1999. Twenty-eight food items were recorded composing the fish diet, being insects the main dietary items. There were also recorded Acari, micro-crustaceans (Cladocera and Copepoda), Aracnida and Crustacea shrimps, fish scales and seeds. It was concluded that in Camaleão Lake, $T$. flavus when juvenile, is an omnivorous fish with a wide feeding spectrum, and its diet is mainly composed of invertebrates such as Formicidae and Homoptera.
\end{abstract}

KEYWORDS: Triportheus flavus, diet, food items, insects, Camaleão Lake. 


\section{INTRODUÇÃo}

Triportheus flavus (Cope, 1872), também conhecido pela sinonimia T. angulatus (Spix \& Agassiz, 1829), é um peixe caracídeo que pertence a um gênero amplamente distribuído na América do Sul, sendo registrados até 15 espécies. Os peixes desse gênero são encontrados desde a Guiana Francesa até a bacia do rio da Prata na Argentina, ocupando uma ampla diversidade de habitats (Planquette et al., 1996). Na bacia amazônica o gênero Triportheus está representado por seis espécies: Triportheus flavus, $T$. pictus, T. albus, T. elongatus, T. culter e T. curtus. Ferreira et al. (1998) descreve T. flavus como uma espécie de médio porte, que alcança $25 \mathrm{~cm}$ quando adulto e que caracteriza-se pelo corpo alto e comprimido lateralmente, apresentando uma coloração cinza-escura. Na Amazônia Central, esta espécie é migradora, de reprodução total e sem proteção da prole e que constitui um importante recurso aproveitável pelas pescarias da região (Batista \& Petrere-Junior, 2003). Assim, Ferreira et al. (1998) registra que $T$. flavus, T. elongatus e T. albus são espécies de importância comercial do médio Amazonas.

Alguns estudos têm mostrado que espécimes do gênero Triportheus apresentam mecanismos para tolerar baixas concentrações de oxigênio e até mesmo condições de anoxia, fenômeno que geralmente acontece às noites nos lagos de várzea amazônicos (Junk et al., 1983; Soares, 1993). Esta adaptação fisiológica importante provavelmente ajudou para a grande dispersão desse gênero nos mais diversos ecossistemas de nosso continente. Quando jovens, são encontradas associados a bancos de macrófitas aquáticas nos diversos lagos da região, se alimentando de pequenos invertebrados (Sánchez-Botero et al., 2007) e procurando protecao dos peixes predadores como por exemplo Pellona flavipinnis (Moreira-Hara et al., 2009), mas quando adultas podem colonizar as extensas áreas de floresta alagada para aproveitar principalmente a grande diversidade de frutos e sementes que esses ambientes oferecem para a fauna visitante no período de enchente dos rios amazônicos (Goulding, 1980; Almeida, 1984; Braga, 1990; Petry et al., 2003; Marín \& Aron, 2006).

As macrófitas flutuantes constituem um hábitat vital para muitos peixes das várzeas. Crescem geralmente nas margens de lagos e canais (paranás) e são compostas geralmente pelas gramíneas Paspalum repens e Echinochloa polystachya e por plantas flutuantes menores como Eichornia crassipes, Pistia stratiotes e Salvinia spp. (Queiroz \& Crampton, 1999; Sánchez-Botero et al., 2001, 2003). A ictiofauna residente nos capinzais é composta por várias espécies de importância ornamental. Incluso, uma espécie desse gênero Triportheus elongatus forma parte da lista brasileira de peixes ornamentais registrados junto ao IBAMA(Chao, 1998).

Porém, apesar da diversidade, abundância, e importância que estes peixes têm na cadeia trófica dos ambientes lacustres tropicais, sejam como transportadores de energia para os piscívoros, ou bem como espécies que poderiam ser exploradas na pescaria ornamental, pouco se sabe acerca de sua biologia. O presente trabalho é uma contribuição ao conhecimento da biologia alimentar de Triportheus flavus no Lago Camaleão, um típico lago de várzea da Amazônia Central.

\section{MATERIAL E MÉTODO}

\section{ÁREA DE ESTUDO}

O estudo foi realizado no Lago Camaleão, um lago de várzea localizado a $3^{\circ} 15^{\prime} 12^{\prime \prime} \mathrm{S}$ e $59^{\circ} 57^{\prime} 37^{\prime \prime} \mathrm{W}$ na Ilha de Marchantaria, que é uma típica ilha de várzea localizada no rio Solimões-Amazonas, numa área pertencente ao município de Iranduba, a cerca de 15 $\mathrm{km}$ do encontro das águas (Figura 1).

Segundo Irion et al. (1983), esta Ilha foi formada pela continua deposição de sedimentos do próprio rio Amazonas, sendo periodicamente inundada, de maio a junho; quando o nível da água aumenta em média de 10 metros em relação à época de águas baixas que é de novembro a janeiro; período em que a ilha fica quase isolada, e seus vários lagos ficam com profundidades mínimas $(0.5 \mathrm{~m}-1.0 \mathrm{~m})$. O Lago Camaleão é longo e estreito, com aproximadamente $6.5 \mathrm{~km}$ de comprimento e 300 a $500 \mathrm{~m}$ de largura.

\section{COLETAS DE CAMPO}

Os peixes foram coletados durante o mês de Abril de 1999 (período de cheia) das zonas com abundantes macrófitas aquáticas utilizando-se redinhas de cerco de $10 \mathrm{~m}$ de comprimento, $2 \mathrm{~m}$ de altura e $15 \mathrm{~mm}$ de abertura de malha. Após a captura, os peixes foram colocados em sacos plásticos, fixados com solução de formalina ao $10 \%$ e transportados até o Laboratório de Plâncton do INPA em Manaus, aonde foram triados e identificados segundo a chave publicada por Ferreira etal. (1998).

\section{ANÁLISEDA DIETA}

O trabalho foi baseado nas análise do conteúdo estomacal de um total de 50 exemplares juvenis de Triportheus flavus (55.4 $9.9 \mathrm{~mm} \mathrm{CP}$ ). Os conteúdos estomacais foram retirados utilizando material cirúrgico e conservados em solução de formalina ao $8 \%$ em frascos de vidro individualizados e 
etiquetados. Para o estudo da dieta utilizou-se os métodos da freqüência de ocorrência $(F O i)$ e do Volume $(V i)$ os quais foram relacionados a fim de determinar-se, a importância relativa de cada item na dieta dos peixes, mediante o Índice Alimentar de Kawakami-Vazzoler (1980), baseado na seguinte fórmula:

$$
\mathrm{Iai}=\frac{F \boldsymbol{O}_{i} \cdot V_{i}}{\sum F \boldsymbol{O}_{i} \cdot V_{i}}
$$

Para serem usados nesta fórmula, a freqüência de ocorrência $F O i$ foi a relação entre o número de estômagos com determinado item " $i$ " e o número total de estômagos, e o volume $V i$ foi a estimativa visual do volume de cada item em relação ao volume total presente em cada amostra segundo o método de Bemvenute (1990) que consiste em colocar o conteúdo em uma placa Petri, não ultrapassando $1 \mathrm{~mm}$ de espessura, seguida da determinação da área total ocupada por cada item, através de um papel milimetrado colocado sob a placa Petri.

A determinação dos tipos de alimentos encontrados foi feita após uma verificação inicial da sua natureza, tendo em consideração as seguintes categorias:
- Cladocera: micro-crustáceos que pelos seus movimentos são denominados pulgas d'água.

- Copepoda: micro-crustáceos de corpo alongado dividido em cabeça, tórax e abdome.

- Insecta: tanto larvas como adultos de diversas ordens da Classe Insecta.

- Camarões: pequenos crustáceos do ordem Decapoda.

- Acaros: organismos pertencentes ao grupo Acarina

- Aranhas: invertebrados pertencentes ao grupo Araneae.

- Escamas de peixes: geralmente de espécies indeterminadas.

- Restos vegetais: sementes de frutas não identificadas e/ou restos vegetais.

- Material semi-digerido: material difícil de ser identificado por estar em avançado processo de digestão.

A identificação dos organismos zooplanctônicos foi feita até o nível taxonômico mais específico possível, usando-se chaves especializadas (Brandorff et al., 1982; Korinek et al., 1997; Korovchinsky, 1992; Smirnov, 1992; Elmoor-Loureiro, 1997; entre outros). A identificação dos insetos foi feita por especialistas no Laboratório de Entomologia do INPA.

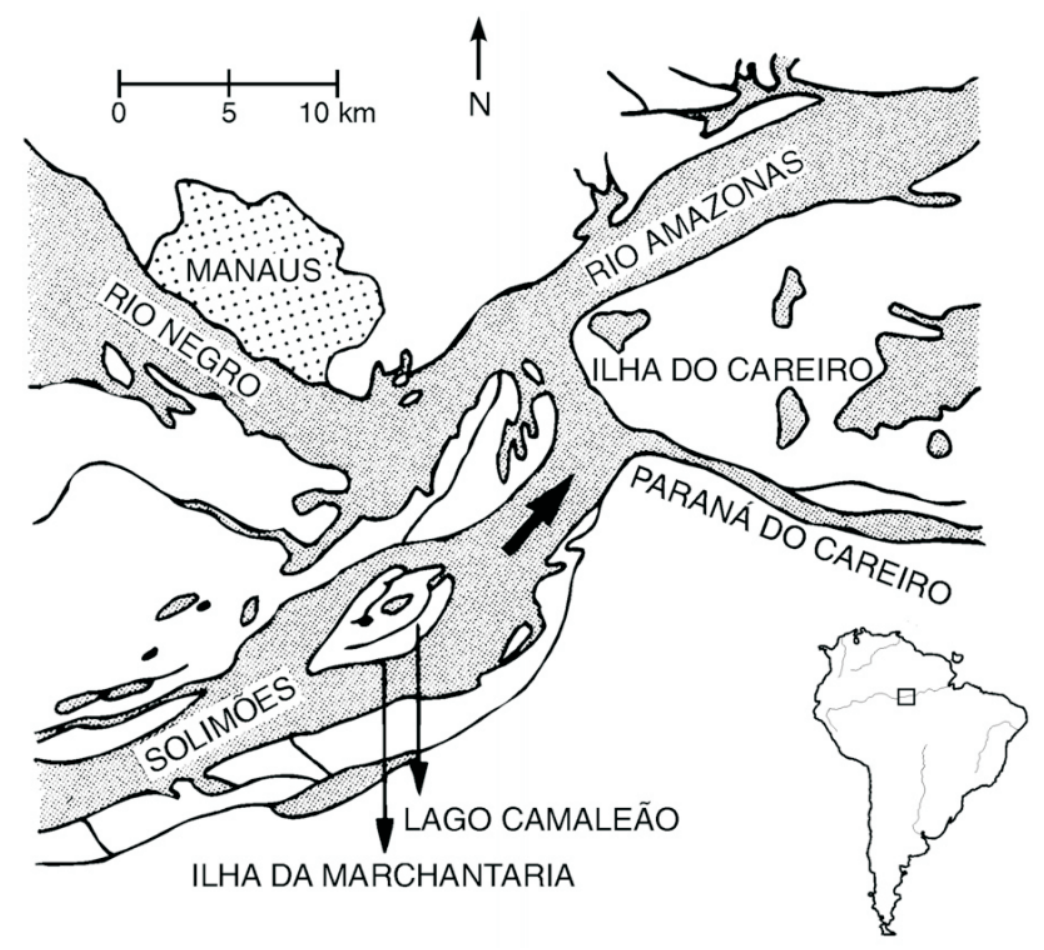

Figura 1. Localização do Lago Camaleão na ilha da Marchantaria en el Estado de Amazonas, Brasil (Furch etal., 1983) 


\section{RESULTADOS E DISCUSSÃO}

Na Tabela 1, pode-se observar os itens alimentares registrados na dieta desta espécie. Observa-se, que os insetos e os cladóceros são os itens mais diversos na dieta nesse período do ano. Sem dúvida, impressiona a diversidade de insetos que esta espécie é capaz de incluir na sua dieta, desde insetos terrestres até aqueles com algum estágio aquático como as larvas de Diptera. Entretanto, doze espécies de cladóceros também formaram parte da dieta, mostrando que também podem aproveitar recursos que sejam abundantes no ambiente em determinado momento, concordando com a teoria de forrageamento ótimo (Townsend \& Winfield, 1985).

Os juvenis de T. flavus parecem ser peixes oportunistas e muito ativos, pois têm a capacidade de capturar diversos tipos de presas, entre micro-crustáceos, larvas e insetos adultos e até camarões, que são organismos que possuem tamanhos, habitats e movimentos diferenciados. A maioria dos invertebrados como insetos, zooplâncton e camarões; são importantes fontes alimentares para os peixes habitantes dos capinzais. Outro fato que condiciona a predação desses organismos é sua grande diversidade, tanto em espécies, tamanhos, formas e distribuição espaço-temporal nos ambientes lacustres.

Segundo Junk (1970, 1973), os capins flutuantes contêm uma rica fauna de invertebrados autóctones, $o$ qual hipoteticamente sustentaria a ictiofauna concentrada nesses lugares. Porém, Zaret (1984) e Chu-Koo (2000), observaram que os pequenos peixes residentes nos capinzais migram às águas abertas em determinadas horas do dia para explorar o zooplâncton desses ambientes. Desse modo, observou-se na dieta de T. flavus organismos próprios da zona litoral como cladóceros não planctônicos (ex. Chydorus sphaericus e Camptocercus dadayi) mas também itens próprios de águas abertas como $D$. spinulosum e $M$. minuta.

Na Tabela 1 também pode-se observar a importâcia relativa de cada item na dieta do peixe, representados pelo índice de Kawakami-Vazzoler. Assim, vemos que os itens mais importantes para os juvenis de T. flavus no lago Camaleão foram os insetos (IAi $=0.698)$, representados principalmente pelas formigas, fato que também foi encontrado por Yamamoto et al. (2004) na mesma espécie. Apesar de sua dieta abrangente, aparentemente, esta espécie de sardinha poderia ser classificada de insetívora nesse período do ano. Desse modo, os juvenis de $T$. flavus parecem mais especializados na captura de insetos, principalmente formigas, as quais provavelmente capturam das folhas das macrófitas flutuantes ou quando estes invertebrados caem nas águas do lago por efeito do vento. A ocorrência dos Aleyrodidae e
Entomobryidae, deve-se a que estes insetos são provavelmente capturados das raízes das macrófitas aquáticas.

Num aspecto geral, Almeida (1984) em ciclo hidrológico semelhante ao nosso, mas no Lago Castanho (Amazonas, Brasil), já tinha observado este comportamento alimentar em exemplares adultos de T. angulatus, verificando que os insetos também foram itens muito freqüentes; mas a composição da dieta em peixes adultos é um pouco diferente, tendo encontrado uma grande proporção de vegetais superiores, alguns Orthoptera e algas. Entretanto num lago de várzea da Amazônia peruana, Marin \& Aron (2006) indicaram que a dieta de exemplares de T. angulatus entre 14 a 20 $\mathrm{cm}$ de comprimento total na época de cheia, esteve composta principalmente por material vegetal procedente das áreas alagáveis, como sementes e flores de palmeras do gênero Bactris, ficando demonstrado a enorme plasticidade alimentar desta espécie. Por outro lado, Catella (1992) na Baía da Onça observou que a dieta de Triportheus sp. consistia basicamente de insetos terrestres, micro-crustáceos e vegetais superiores mas no período de seca no rio Aquidauana (Brasil).

Quanto à significativa presença de cladóceros na dieta e a ausência de outros organismos como os rotíferos, Fregadolli (1993) comenta que dentre os organismos zooplanctônicos, os cladóceros são mais atraentes visualmente do que outras presas, o qual explicaria sua significativa presença na dieta desse peixe. Aparentemente, os cladóceros não planctônicos que foram registrados na dieta, foram capturados apartir do perifiton adherido às raízes das macrófitas aquáticas do lago. É importante destacar o consumo de cladóceros devido a que um estudo similar publicado por Yamamoto et al. (2004) reporta que a dieta dos juvenis de esta espécie (aprox. $4-8 \mathrm{~cm}$ ) foi básicamente composta de insetos, nao reportando nenhum tipo de organismo zooplantônico no trato digestivo de T. flavus no Lago Camaleao, o qual difiere completamente com os nossos resultados.

Devido à sua ampla distribuição geográfica no continente americano, as espécies deste gênero podem apresentar diferencias na composição de suas dietas, segundo o habitat onde ocorrem. As sardinhas geralmente são espécies onívoras que aproveitam os items alimentares mais abundantes na temporada, como já foi observado em T. albus (Ferreira et al., 1998); T. elongatus (Almeida, 1984), T. paranensis, $T$. nematurus (Galina \& Hahn, 2003), T. signatus (Höfling et al., 2000) e T. angulatus (Braga, 1990; Yamamoto et al., 2004; Marín \& Aron, 2006); mas pode haver algumas exceções como na espécie $T$. culter que é exclusivamente zooplanctófaga (Almeida, 1984; Chu-Koo, 2000). 
Embora as macrófitas aquáticas não foram uma importante fonte de alimento para a sardinha, seus caules e raízes funcionam como substrato para o perifíton (Engle \& Melack, 1990; Caffrey, 1993) e abrigam ainda, uma grande quantidade de invertebrados (Junk, 1973; Blanco-Belmont, 1990;
Junk \& Robertson, 1997), que constituem importantes itens alimentares para os juvenis de Characiformes migradores (Goulding \& Carvalho, 1982), incluindo muitas espécies explotadas pela frota pesqueira da Amazônia (Sánchez-Botero \& Araújo-Lima, 2001) como neste caso, T. flavus.

Tabela 1. Itens alimentares registrados na dieta de juvenis de Triportheus flavus do Lago Camaleão (Amazonas, Brasil) na época de enchente.

\begin{tabular}{|c|c|c|c|c|}
\hline ITENS ALIM & FOi (\%) & VOi (\%) & FOi x VOi & $\mathbf{I A i}$ \\
\hline Cladocera & 26.0 & 12.7 & 313.42 & 0.072 \\
\hline Bosmina hagmanni & 26.0 & 0.2 & 5.2 & 0.002 \\
\hline Camptocercus dadayi & 26.0 & 0.6 & 16.6 & 0.003 \\
\hline Ceriodaphnia cornuta & 10.0 & 0.1 & 0.5 & ---- \\
\hline Chydorus sphaericus & 26.0 & 0.3 & 7.8 & 0.002 \\
\hline Daphnia gessneri & 18.0 & 0.5 & 8,28 & 0.002 \\
\hline Diaphanosoma brevireme & 26.0 & 0.1 & 1.3 & ---- \\
\hline Diaphanosoma polyspina & 26.0 & 1.7 & 43.16 & 0.010 \\
\hline Diaphanosoma spinulosum & 26.0 & 8.7 & 225.68 & 0.050 \\
\hline Macrothrix goeldii & 10.0 & 0.1 & 1.2 & ---- \\
\hline Macrothrix triserialis & 10.0 & 0.1 & 0.8 & ----- \\
\hline Moina minuta & 26.0 & 0.3 & 7.8 & 0.002 \\
\hline Simocephalus sp. & 26.0 & 0.2 & 4.16 & 0.001 \\
\hline Insecta & 78.0 & 62.9 & 3160.2 & 0.698 \\
\hline Aleyrodidae & 78.0 & 22.3 & 1740.2 & 0.385 \\
\hline Culicidae (pupas) & 12.0 & 3.4 & 41.2 & 0.010 \\
\hline Chaoboridae (larvas) & 18.0 & 1.1 & 19.4 & 0.005 \\
\hline Chironomidae (larvas) & 18.0 & 0.6 & 10.3 & 0.002 \\
\hline Dytiscidae & 6.0 & 1.7 & 10.3 & 0.002 \\
\hline Entomobryidae & 25.0 & 7.2 & 178.8 & 0.039 \\
\hline Formicidae & 62.0 & 16.4 & 1016.2 & 0.224 \\
\hline Helodidae & 12.0 & 3.4 & 41.2 & 0.009 \\
\hline Hemiptera & 18.0 & 5.1 & 92.5 & 0.020 \\
\hline Lepidoptera & 6.0 & 1.7 & 10.3 & 0.002 \\
\hline Copepoda & 5.0 & 0.04 & 0.20 & --- \\
\hline Aracnida & 6.0 & 0.37 & 2.22 & ---- \\
\hline Acarina & 10.0 & 2.9 & 28.6 & 0.006 \\
\hline Camarões & 10.0 & 2.8 & 28.0 & 0.006 \\
\hline Peixes (escamas) & 50.0 & 5.83 & 291.5 & 0.064 \\
\hline Restos vegetais (sementes) & 8.0 & 5.3 & 95.2 & 0.021 \\
\hline Material semi-digerido & 85.0 & 7.1 & 603.5 & 0.133 \\
\hline
\end{tabular}




\section{CONCLUSÕES}

Os insetos e outros invertebrados associados a macrófitas litorâneas como os cladóceros e camarões por sua grande disponibilidade no ambiente, diversificação de formas, de tamanhos e movimentos são as mais importantes fontes alimentares para os juvenis de T. flavus do Lago Camaleão no período de cheia e contribuem significativamente à passagem do fluxo de energia dos produtores e primeiros elos da cadeia alimentar para os peixes, disponibilizando a entrada dessa energia à cadeia trófica dos ecossistemas lacustres de várzea. De fato, se na dieta do peixe estiveram contemplados vários itens próprios das águas abertas, suspeita-se que esta espécie residente do capinzal, provavelmente migra às águas abertas para melhorar sua dieta com o zooplâncton, fato já observado em outros peixes amazônicos.

Conclui-se, segundo a composição de sua dieta que, $T$. flavus e um peixe onívoro com forte tendência à insetivoria no estagio juvenil, e que tem um comportamento alimentar sumamente oportunista.

\section{AGRADECIMENTOS}

Ao CNPq pela ajuda financeira através das Bolsas de Mestrado e Doutorado para tres autores do presente trabalho. Ao Instituto Nacional de Pesquisas da Amazônia (INPA) pelas facilidades logisticas (uso de equipos e materiais de laboratório, apoio nas amostragens de campo, etc.).

\section{BIBLIOGRAFIA CITADA}

Almeida, R.G. 1984. Biologia alimentar de três espécies de Triportheus (Pisces:

Characidae) do lago do Castanho, Amazonas. Acta Amazônica, 14 (1-2): 48-76.

Batista, D. V.; Petrere-Junior, M. 2003. Characterization of the comercial fish production landed at Manaus, Amazonas State, Brazil. Acta Amazonica, 33(1): 53-66.

Bemvenute, M. A. 1990. Hábitos alimentares de peixes rei (Atherinidae) na região estuarina da Lagoa dos Patos. Atlantica, 12: 79 - 102.

Blanco-Belmont, L. 1990. Estudio de las comunidades de invertebrados associados a las macrofitas acuaticas de tres lagunas de inundacion de la seccion baja del Orinoco. Sociedad de Ciencias Naturales La Salle, Tomo IL-L: 71-107.

Braga, F. M. 1990. Aspectos da reprodução e alimentação de peixes comuns en um trecho do rio Tocantis entre Imperatriz e Estreito, Estados de Maranhão e Tocantis, Brasil. Revista Brasileira de Biologia, 50 (3): 547-558.
Brandorff, G. -O.; Koste, W.; Smirnov, N. N. 1982. The composition and structure of Rotiferan and Crustacean communities of the lower Rio Nhamundá, Amazonas, Brazil. Stud. Neotrop. Fauna Environ., 17: 69-121.

Caffrey, J. M. 1993. Aquatic plant management in relation to Irish recreational fisheries development. Journal of Aquatic Plant Management, 31: 162-168.

Catella, A. C. 1992. Estrutura da comunidade e alimentação dos peixes da Baía da Onça, uma lagoa do Pantanal do rio Aquidauana, MS. Dissertação de Mestrado em Ciências. Universidade de Campinas. Campinas, SP. Brasil. $125 \mathrm{p}$.

Chao, N. L. 1998. A draft List of brazilian freshwater fishes for the hobby: a proposal to IBAMA. OFI Journal, 23: 6-14

Chu-Koo, F. W. 2000. Interações peixes/zooplâncton no Lago Camaleão, um lago de várzea da Amazônia Central, Brasil. Dissertação de Mestrado. Instituto Nacional de Pesquisas da Amazônia/Fundação Universidade do Amazonas. Manaus, Amazonas, Brasil. 91p.

Elmoor-Loureiro, L.M. 1997. Manual de identificação de cladóceros límnicos do Brasil. Brasili: Universa, Brasília, DF. 156p.

Engle, D. L.; Melack, J. M. 1990. Floating meadow epiphyton: Biological and chemical features of epiphytic material in an Amazon floodplain lake. Freshwater Biology, 23: 479-494.

Ferreira, E. J. G.; Zuanon, J. A. S.; Dos Santos, G. M. 1998. Peixes comerciais do médio Amazonas: Região de Santarém, Pará. Edições IBAMA, Brasília. 214p.

Fregadolli, C. H. 1993. Selecao alimentar das larvas de pacu Piaractus mesopotamicus Holmberg, 1887 e tambaqui Colossoma macropomum Cuvier, 1818 em laboratório. Boletim Técnico CEPTA, 6(1): 150.

Furch, K.; Junk, W.J.; Dietrich, J.E.; Kochert, N. 1983. Seasonal variation in the major cation $(\mathrm{Na}, \mathrm{K}, \mathrm{Mg}$ and $\mathrm{Ca}$ ) content of the water of Lago Camaleão, an Amazonian floodplain lake near Manaus, Brazil. Amazoniana, 8 (1): 75-89.

Goulding, M. 1980. The fishes and the forest Explorations in Amazonian Natural History. University of California Press, Los Angeles, CA, USA. 280p.

Goulding, M.; Carvalho, M. 1982. Life history and management of the tambaqui (Colossoma macropomum, Characidae): An important Amazonian food fish. Revista Brasileira de Zoologia, 1: 107-133. 
Irion, G.; Adis, J. Junk, W.J.; Wunderlich, F. 1983. Sedimentological studies of the "Ilha de Marchantaria" in the Solimões/Amazon river near Manaus. Amazoniana, 8 (1):1-18.

Junk, W. J. 1970. Investigations on the ecology and production - biology of the "floating meadows" (Paspalo -Echinochloetum) on the Middle Amazon. Part I. The floating vegetation and its ecology. Amazoniana, 2: 449-495.

Junk, W. J. 1973. Investigations on the ecology and production - biology of the "floating meadows" (Paspalo-Echinochloetum) on the Middle Amazon. Part II. The aquatic fauna in the root zone of the floating vegetation. Amazoniana, 4: 9-102.

Junk, W. J.; Soares, G. M.; Carvalho, F. M. 1983. Distribuition of fishes species in a lake of the Amazon river floodplain Manaus (Lago Camaleão), with special reference to extreme oxygen conditions. Amazoniana, 7 (4):397-431.

Junk, W. J.; Robertson, B. 1997. Aquatic invertebrates. In: Junk, W. J. (Ed.). The Central Amazonian floodplain: Ecology of a pulsing system. Ecological Studies, vol. 126. Springer Verlag, Heidelberg, Germany, p.279-298.

Kawakami, E.; Vazzoler, G. 1980. Método gráfico e estimativa de índice alimentar aplicado no estudo de alimentação de peixes. Bol. Instit. Oceanogr. S. Paulo, 29 (2): 205-207.

Korinek, V.; Sacherova, V.; Havel, L. 1997. Subgeneric differences in head shield and ephipia ultrastructure within the genus Bosmina Baird (Crustacea, Cladocera). Hydrobiologia, 360:1323.

Korovchinsky, N. M. 1992. Sididae and Holopedidae:(Crustacea, Daphniformes). The Hague: SPB Academic Publishing. 82 p.

Marin, E. G.; Aron, N. A. 2006. Variación temporal de la dieta de sardina Triportheus angulatus (Characiformes, Characidae) en una laguna de inundación de la selva amazónica peruana. Folia Amazónica, 15(1-2): 141-150.

Moreira-Hara, S. S.; Zuanon, J. A. S.; Amadio, S. A. 2009. Feeding of Pellona flavipinnis (Clupeiformes, Pristegasteridae) in a Central Amazonia floodplain. Iheringia Série Zoologia, 99(2): 153-157.
Petry, P.; Bayley, P. B.; Markle, D. F. 2003. Relationships between fish assemblages, macrophytes and environmental gradients in the Amazon River floodplain. Journal of Fish Biology, 63 (3): 547-579.

Planquette, P.; Keith, P. ; Le Bail, P-Y. 1996. Atlas des poissons d'eau douce de Guyane (tome 1). Collection du Patromoine Naturel, Vol 22. IEGBM.N.H.N., INRA, CSP, Min. Env., Paris, 429p.

Queiroz, H. L.; Crampton, W. G. R. 1999. Estratégias para manejo de recursos pesqueiros em Mamirahuá. Helder L. Queiroz, William G. R. Crampton Eds. Brasília: Sociedade Civil Mamirahuá. CNPq. 208 p.

Sánchez-Botero, J. I.; Araújo-Lima, C. A. R. M. 2001. As macrófitas aquáticas como berçário para a ictiofauna da várzea do rio Amazonas. Acta Amazonica, 3 (3): 437-447.

Sánchez-Botero, J. I.; Farias, M. L.; Piedade, M. T.; Garcez, D. S. 2003. Ictiofauna associada às macrófitas aquáticas Eichhornia azurea (SW.) Kunth. e Eichhornia crassipes (Mart.) Solms. no lago Camaleão, Amazônia Central, Brasil. Acta Scientiarum Biological Sciences, 25 (2): 369-375.

Sánchez-Botero, J. I.; Leitão, R. P.; Caramaschi, E. P.; Gracez, D. S. 2007. The aquatic macrophytes as refuge, nursery and feeding habitats for freshwater fish from Cabiúnas lagoon, restinga de Jurubatiba National Park, Rio de Janeiro, Brazil. Acta Limnológica Brasileira, 19 (2): 143-153.

Smirnov, N. N. 1992. The Macrothricidae of the world. III (Guides to the identification of the macroinvertebrates of the continental waters of the world). The Hague: SPB Academic Publishing. $143 \mathrm{p}$.

Townsend, C. R.; Winfield, I. J. 1985. The application of optimal foraging theory to feeding behaviur in fish. (In): Tyler, P. T. \& Calow, P. (eds.). Fish Energetics: New Perspectives. pp. 67-98. Baltimore: Johns Hopkins Univ. Press.

Yamamoto, K. C.; Soares, M. G. M.; Freitas, C. E. C. 2004. Alimentação de Triportheus angulatus (Spix and Agassiz, 1829) no lago Camaleão, Manaus, AM, Brasil. Acta Amazonica, 34(4):653-659.

Zaret, T. M. 1984. Interactions fish/ zooplankton in amazon floodplains lakes. Verh. Internat. Verein. Limnol., 22: 1305-1308. 


